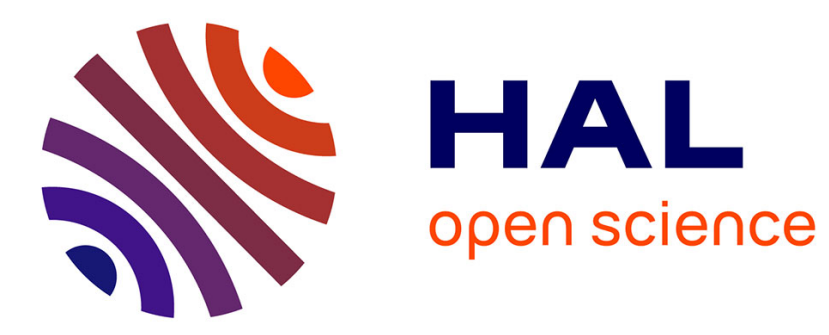

\title{
L-Stable parallel one-block methods for ordinary differential equations
}

Philippe Chartier, Bernard Philippe

\section{To cite this version:}

Philippe Chartier, Bernard Philippe. L-Stable parallel one-block methods for ordinary differential equations. [Research Report] RR-1650, INRIA. 1992. inria-00074910

\section{HAL Id: inria-00074910 \\ https://hal.inria.fr/inria-00074910}

Submitted on 24 May 2006

HAL is a multi-disciplinary open access archive for the deposit and dissemination of scientific research documents, whether they are published or not. The documents may come from teaching and research institutions in France or abroad, or from public or private research centers.
L'archive ouverte pluridisciplinaire HAL, est destinée au dépôt et à la diffusion de documents scientifiques de niveau recherche, publiés ou non, émanant des établissements d'enseignement et de recherche français ou étrangers, des laboratoires publics ou privés. 


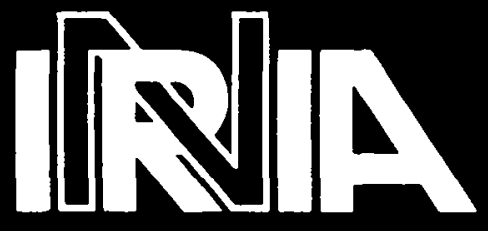

UNITÉ DE RECHERCHE INRIA-RENNES

Institut National de Recherche en Informatique et en Automatique

Domaine de Voluceau Rocquencourt B.P.105

78153 LeChesnay -Cedex France

Tél::(1) 39635511

\section{Rapports de Recherche}

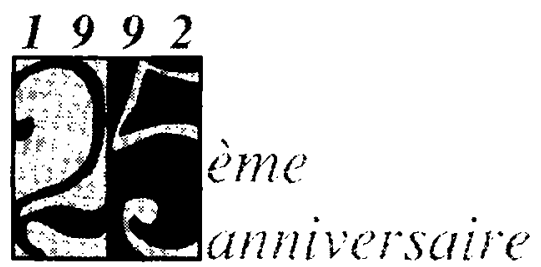

$\mathrm{N}^{\circ} \mathbf{1 6 5 0}$

\section{Programme 1}

Architectures parallèles, Bases de données, Réseaux et Systèmes distribués

\section{L-STABLE PARALLEL ONE-BLOCK METHODS FOR ORDINARY DIFFERENTIAL EQUATIONS}

\section{Philippe CHARTIER} Bernard PHILIPPE 
Campus Universitaire de Beaulieu 35042 - RENNES CEDEX FRANCE

Tèl. : 998471 00 - Télex : UNIRISA $950473 \mathrm{~F}$

Télécopie : 99383832

\title{
L-Stable Parallel One-Block Methods for Ordinary Differential Equations
}

\author{
Philippe Chartier*, Bernard Philippe ${ }^{\dagger}$
}

Programme 6

Publication interne $\mathrm{n}^{0} 632-28$ pages.

Janvier 1992

\section{Méthodes à un bloc L-stables \\ Résumé}

Nous considérons ici les méthodes à un bloc développées par W. Couzy, B.P. Sommeijer et P.J. van der Ilouwen. Ces méthodes sont destinces à résoudre un système d'équations diff́renticlles ordinaires sur ordinateur parallèle. Nous dérivons de nouvelles conditions d'ordre, ćtudions lcur stalsilité ct exhibons une nouvelle classe de méthodes parallèles. En particulier, nous démontrons qu'clles sont I-stables jusqu'ì l'ordre sept.

Mots-clé : équations différentielles ordinaires, méthodes à un bloc, L-stabilité, ordre ćlevé, parallélisme.

\begin{abstract}
In this contribution, we consider the one-block method designed by W. Couzy, B.P. Sommeijer and P.J. van der Ilouwen for the purpose of solving ordinary differential equations (ODEs) on a parallel computer. We derive a new set of oder conditions, study the stability and exhibit a new class of parallel methods which are proven to be L-stable up to order seven.
\end{abstract}

Key-words : ODEs, one-block methods, L-stability, high order, parallelism.

- SIMULOG, 1 ruc Jaines Joule, 78182 ST QUENTIN YVELINES CEDEX

1 INRIA/IRISA, Cainpus de Beaulicu, 35012 RENNES CEDEX 


\section{Introduction}

The purpose of this paper is to numerically solve the initial value problem

$$
\left\{\begin{array}{l}
y^{\prime}=f(x, y(x)) \\
y\left(x_{0}\right)=y_{0}, x \in\left[x_{0}, X\right]
\end{array}\right.
$$

where $f$ is assumed to be continuous and to satisfy a Lipschitz condition on region $\left[x_{0}, X\right] \times R^{m}$.

In the literature only a few parallel algorithms for solving (1) have been proposed. Generally speaking, speeding up the integration of (1) can be achieved by partitioning the tasks either "across the system of equations" or "across the method", as exemplified by Gear in [5]. In addition to these two types of parallelism, A. Bellen and M. Zennaro have introduced a third one called "across the time" (see [8]). The equation segmentation method is straightforward and widely used by enginecrs in the field of dynamic system simulation. However, its scope is rather limited, since automated partitioning scems feasible only for application-oriented codes in which the structure of $f$ is accessible. Parallelism "across the time" means that each processor evaluates $f$ for different values of $x$. These values are combined into a recurrence that carries the information from the initial point. This kind of parallelism may yield large speedups as far as a large number of processors is available. However, R. Vermiglio reports numerical simulations that suggest severe limitations ${ }^{1}$ (see [9]).

In this paper, we focus on parallelism "across the method". More specifically, we consider parallel block methods. Block methods can be seen as a set of linear multistep methods simultaneously applied to (1) and then combined to yield a "better" approximation. Numerous block methods have been proposed : Shampine and Watts have constructed A-stable implicit one-block methods for very ligh orders (sec [15]), whereas Chu and Hamilton have studied the predictor-corrector formulation of multi-block schemes (see [14]).

More recently, W. Couzy, B.P. Sommeijer and P.J. van der Houwen have extended some of the strong stability properties of the backward differentiation formulae (BDF) (A-stability or $A(\alpha)$ stability) to a new class of parallel one-block methods. Their procedure consists in segmenting the total work per step into a few tasks, so that it actually requires the same amount of work as a sequential execution of a BDF. By numerically scanning the space of free coefficients, they have obtained very promising results with respect to A-stability up to order five. From a slightly different point of view, the search for higher order A-stable multistep methods has been carried out in two directions : the use of second derivative of the solution (see $[12,13,6,7]$ ) and the study of new "general linear methods" (see [10]). These methods have a better numerical behavior (A-stability" up to order six) but are fully implicit and consequently not appropriate for parallel computers.

Our purpose is to carry on the work of [4] and more specifically to construct high-order A-stable schemes that are furthermore easy to implement on a parallel computer. In section 2, we recall the definition of parallel implicit one-block method introduced in [4] and we derive a new set of order conditions. Besides, we apply some fairly classic results on stability to get a practical criterium for A-stability.

Section 3 is devoted to the two-processor case. The use of symbolic calculus gave us an opportunity to handle rather complicated formulae and therefore to construct a L-stable two-processor method of order three.

Section 4 discusses a rather different approach. The complexity of order and stability conditions have led us to leave the frightening jungle of symbolic calculus (at least for a moment). Rather than trying to solve this large number of algebraic conditions, we designed a new class of methods by making strong simplifying assumptions. These methods exhibited surprisingly good stability properties for very high orders. An analytical proof of their L-stability up to order seven has been given. Evidence of L-stability for orders eight and nine are reported, while it is likely that the methods remain L-stable for higher orders (we did not find any analytical proof).

\footnotetext{
${ }^{1}$ Actually, this approach seems feasible only for equations which solution does not depend too strongly on the initial condition (see [11]).
} 
Finally, section 5 reports result.s of accuracy tests and stability tests. These two sets of tests are in perfect agreement with the theoretical results.

\section{Parallel One-Block Methods}

We present a class of methods, introduced by B.P. Sommeijer, W. Couzy and P.J. van der Houwen (see [4]), which are a direct generalization of the implicit one-step method :

$$
y_{n+1}=a \cdot y_{n}+h \cdot b \cdot f\left(y_{n}\right)+h \cdot d \cdot f\left(y_{n+1}\right)
$$

as well as a restriction of the multi-block methods of M.T. Chu and H. Hamilton (see [14]).

\subsection{Definition}

Let us introduce the following vector :

$$
c=\left(c_{1}, \cdots c_{k}\right)^{T}
$$

with either $c_{1}=1$ or $c_{k}=1$ (the $c_{i}^{\prime} s$ are assumed to be pairwise distinct), and let $y_{n, i}$ denote a numerical approximation of the exact solution value $y\left(x_{n}+c_{i} . h\right)$ and $Y_{n}$ denote vector $\left(y_{n, 1}^{T}, \cdots, y_{n, k}^{T}\right)^{T}$. We then give the following definition:

Definition 1 We call a $k$-dimensional parallel block method, a method defined by the recursion :

$$
Y_{n+1}=(A \otimes I) Y_{n}+h \cdot(B \otimes I) F\left(Y_{n}\right)+h \cdot(D \otimes I) F\left(Y_{n+1}\right)
$$

where $A, B$ and $D$ are $k$-by-k real matrices and $D$ is assumed to be diagonal.

According to the usual convention, for any given vector $v=\left(v_{1}, \cdots, v_{k}\right)^{T}, F(v)$ denotes the vector $\left(f\left(v_{1}\right), \cdots, f\left(v_{k}\right)\right)^{T}$. Let us emphasize that $D$ is diagonal, so that it is possible to decouple the various components of the solution : if $k$ processors are used, each can solve a system of equations with Jacobian matrix $I_{m}-h \cdot D_{i, i} \cdot \frac{\partial f}{\partial y}$. We can also notice that $c$ is allowed to have $k-1$ noninteger components. The first or last component of $Y_{n+1}$ will be retained as the numerical approximation of the solution at point $x_{n+1}$

\subsection{Formulation as a "general linear method"}

The methods previously defined can be easily recasted into the class of Butcher's "general linear methods" (see [3]). Following the presentation of Hairer, Wanner and Norsett (see [1] p.386), we may define a forward step procedure, a correct value function and a starting procedure as follows :

Forward step procedure :

$$
U_{n}=\left(\begin{array}{c}
Y_{n} \\
h . F\left(Y_{n}\right)
\end{array}\right),\left\{\begin{array}{c}
U_{n+1}=\left(\left(\begin{array}{cc}
A & B \\
0 & 0
\end{array}\right) \otimes I\right) U_{n}+h\left(\left(\begin{array}{c}
D \\
I
\end{array}\right) \otimes I\right) F\left(V_{n}\right) \\
V_{n}=\left(\left(\begin{array}{cc}
A & B
\end{array}\right) \otimes I\right) \quad U_{n}+h \quad(D \otimes I) \quad F\left(V_{n}\right)
\end{array}\right.
$$

So that we have:

$$
\mathcal{A}=\left(\begin{array}{cc}
A & B \\
0 & 0
\end{array}\right), \quad \tilde{\mathcal{B}}=D, \quad \tilde{A}=\left(\begin{array}{ll}
A & B
\end{array}\right), \quad \mathcal{B}=\left(\begin{array}{c}
D \\
I
\end{array}\right)
$$


Correct value function:

$$
z(x, h)=\left(\begin{array}{c}
y\left(x+\left(c_{1}-1\right) h\right) \\
\vdots \\
y\left(x+\left(c_{k}-1\right) h\right) \\
h \cdot y^{\prime}\left(x+\left(c_{1}-1\right) h\right) \\
\vdots \\
h \cdot y^{\prime}\left(x+\left(c_{k}-1\right) h\right)
\end{array}\right)
$$

Starting procedure:

$$
U_{0}=\Phi(h)=\left(y_{0}^{T}, y_{1}^{T}, \cdots, y_{k}^{T}, h \cdot y_{1}^{\prime T}, h \cdot y_{2}^{\prime T}, \cdots, h \cdot y_{k}^{\prime T}\right)^{T}
$$

where $\Phi(h)$ approximates $z_{0}=z\left(x_{0}, h\right)$.

According to Skeel's theory, we further define the matrices $S=\mathcal{A}$ and $\mathbb{E}$ for a preconsistent zcrostable method.

Definition 2 A k-dimensional block method with matrix $S$ is called zero-stable if $S^{n}$ is uniformly bounded for all $n$.

Let $T$ be such that the Jordan canonical form of $S$ is :

$$
S=\operatorname{T.diag}\left\{\left(\begin{array}{ccc}
1 & & \\
& \ddots & \\
& & 1
\end{array}\right),\left(\begin{array}{ccc}
\xi_{2} & & \\
& \ddots & \\
& & \xi_{2}
\end{array}\right), \cdots,\left(\begin{array}{ccc}
\xi_{1} & & \\
& \ddots & \\
& & \xi_{l}
\end{array}\right), \tilde{J}\right\} \cdot T^{-1}
$$

where $\xi_{\text {: }}$ are the eigenvalues of $S$ of modulus one. Then, $\mathbb{E}$ is defined as the spectral projector onto the invariant subspace associated with 1 :

$$
\mathbb{E}=T \cdot \operatorname{diag}(I, 0, \cdots, 0) \cdot T^{-1}
$$

\subsection{Reduction to an autonomous system}

Problem (1) may be transformed into the following autonomous system :

$$
\left\{\begin{array}{l}
y^{\prime}=F(Y) \\
Y_{0}=\left(x_{0}, y_{0}^{T}\right)^{T}
\end{array}\right.
$$

where $Y=\left(x, y^{T}\right)^{T} \in \mathbb{R}^{m+1}$ and $F(Y)=\left(1, f(x, y)^{T}\right)^{T}$. When applying a k-dimensional block method to the reformulated problem, we obtain :

$$
\forall i \in[1, \cdots, k], x_{n+1, i}=\sum_{j=1}^{k} a_{i, j} x_{n, i}+h \sum_{j=1}^{k} b_{i, j}+h d_{i}
$$

Equations (3) can be expressed in a vector form by :

$$
x_{n} e+h(c+e)=x_{n} A e+h(A c+B e+D e)
$$

These conditions become :

$$
\left\{\begin{array}{l}
A e=e \\
A(c-e)+B e+D e=c
\end{array}\right.
$$

which imply a consistent method of order $p \geq 1$ and which are satisfied for all methods of order $p \geq 2$, as shown in the next paragraphs. In the sequel, we only consider methods which satisfy (3) and therefore restrict ourselves to the study of $\mathbf{k}$-dimensional block methods on autonomous systems. 


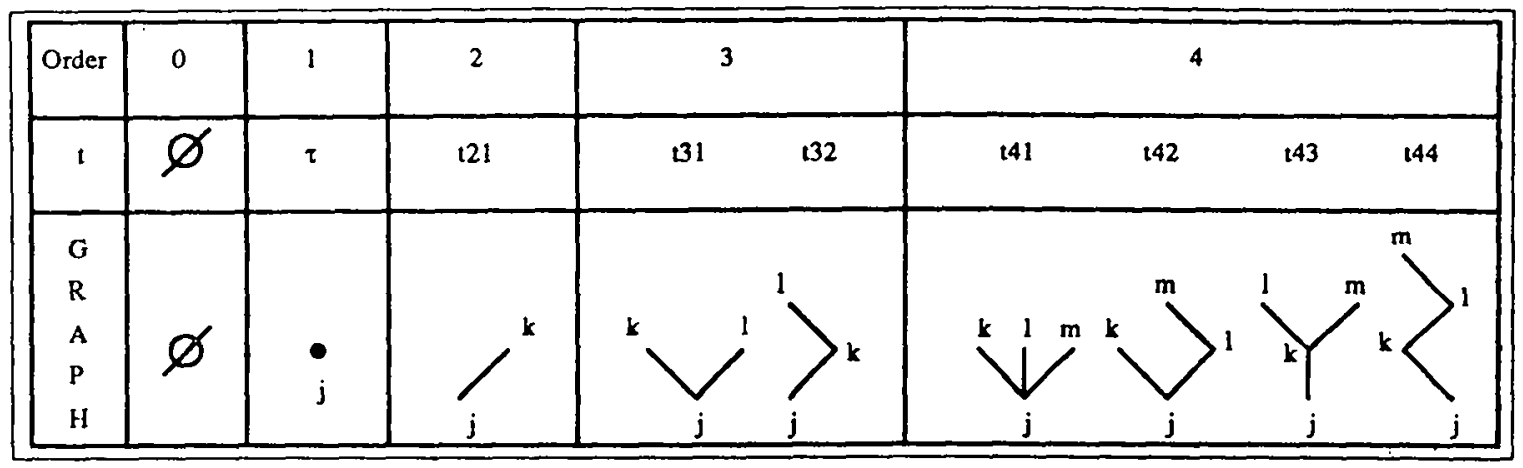

Figure 1: Trees up to order 4

\subsection{Butcher-series}

In order to use Butcher series, we recall a few definitions and notations about trees. For more details, the reader may refer to the book by Hairer, Wanner and Norsett ([1] p. 145) :

Definition 3 Let $C$ be an ordered chain of $q$ indices :

$$
C=\left\{j_{1}<j_{2}<\cdots<j_{q}\right\}
$$

A labelled tree of order $q$ is a mapping $t$ from $C-\left\{j_{1}\right\}$ into $C$, such that $t(r)<r$ for any $r \in C-\left\{j_{1}\right\}$. The order of $t$ is equal to the number of its vertices and is denoted $\rho(t)$. The set of all labelled trees of order $q$ is denoted $L T_{q}$.

Labelled trees that are topologically alike are equivalent and form a class called a tree. The set of all trees of order $q$ is denoted $T_{q}$, while the set of all trees of any order is denoted $T$. We further define $\oslash$ to be the empty tree, $\tau$ to be the only tree of order one and $\tau^{n}$ the $n^{\text {th }}$-order tree recursively defined $\tau^{n}=[\underbrace{\tau, \cdots, \tau}_{n-1}]$ (see fig. 2). For sake of clarity, we give the graph of all trees up to order 4 together with their notations (see fig. (1)). If we denote $y^{J}$ the $j^{\text {th }}$ component of $y$ and assume that (1) is autonomous, then we can associate an elementary differential $F^{J_{1}}$ with each tree $t$ defined on $C=\left\{j_{1}<j_{2}<\cdots<j_{q}\right\}$ :

$$
F^{J_{1}}=\sum_{J_{2}, \cdots, J_{q}} \prod_{i=1}^{i=q} f_{t-1}^{J_{i}\left(J_{i}\right)}
$$

Definition 4 Let $a(\oslash), a(\tau), a\left(t_{21}\right), \cdots$ be a sequence of real coefficients defined for all trees $a: T \longrightarrow$ $\mathbb{R}$. Then we call the series :

$$
\begin{array}{r}
a(\oslash) \cdot y^{J}+h \cdot a(\tau) \cdot f^{J}(y)+\frac{h^{2}}{2 !} \cdot a\left(t_{21}\right) \cdot f_{J}^{K}(y) f^{J}(y)+\cdots \\
=\sum_{t \in L T} \frac{h^{\rho(t)}}{\rho(t) !} a(t) F^{J}(y):=B^{J}(a, y)
\end{array}
$$

a Butcher-series.

Further results will require the expansion of the correct value function as a Butcher-series. We may observe that all coefficients $a(t)$ in the expansion of the correct value function depend only on $\rho(t)$. 
We have indeed:

$$
\begin{array}{r}
y^{J}\left(x+\left(c_{i}-1\right) \cdot h\right)=y^{J}(x)+\left(c_{i}-1\right)\left(y^{J}(x)\right)^{(1)}+\cdots+\frac{\left(c_{i}-1\right)^{n} h^{n}}{n !}\left(y^{J}(x)\right)^{(n)}+\cdots \\
h \cdot\left(y^{J}\left(x+\left(c_{i}-1\right) \cdot h\right)\right)^{(1)}=0+h \cdot\left(y^{J}(x)\right)^{(1)}+\cdots+(n+1) \cdot \frac{\left(c_{i}-1\right)^{n} h^{n+1}}{(n+1) !}\left(y^{J}(x)\right)^{(n+1)}+\cdots
\end{array}
$$

A.cordings to Theorem 2.6. of [1] pp. 146, for the $q^{\text {th }}$ derivative of the true solution, we lave:

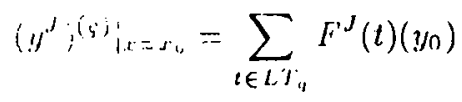

so thisl:

$$
\left\{\begin{array}{l}
a_{i}(\oslash)=1 \\
a_{i}(\tau)=\left(c_{i}-1\right) \\
\vdots \\
a_{i}\left(r^{n}\right)=\left(c_{i}-1\right)^{n}
\end{array}\right.
$$

for all $i$ from 1 to $k$ and

$$
\left\{\begin{array}{l}
a \cdot(0)=0 \\
\therefore \therefore \cdot=1 \\
\vdots \\
a_{i}\left(\tau^{n}\right)=n \cdot\left(c_{i}-1\right)^{n-1}
\end{array}\right.
$$

for all $i$ form $k+1$ to $2 k$.

\section{$2.5 \quad P^{\text {th }}$-order consistency conditions}

We are now able to derive algebraic conditions on the free parameters (the coefficients of $A, B$ and $D$ ) for $p^{\text {th }}$-order consistency. In order to avoid tensor products, from now on we assume that (1) is scalar. whereas all results are obviously valid for systems. However, the definition of a $\mathbf{k}$-dimensional block method makes sense only if preconsistency conditions are satisfied. By denoting $e=(1, \cdots, 1)^{T} \in \mathbb{R}^{k}$, we have here:

$$
z(\Omega)=\left(\begin{array}{c}
e \\
0
\end{array}\right), z(\tau)=\left(\begin{array}{c}
c-e \\
e
\end{array}\right), z\left(\tau^{2}\right)=\left(\begin{array}{c}
(c-e)^{2} \\
2(c-e)
\end{array}\right), \cdots, z\left(\tau^{n}\right)=\left(\begin{array}{c}
(c-e)^{n} \\
n(c-e)^{n-1}
\end{array}\right)
$$

So that :

$$
\mathcal{A} z(\oslash)=z(\oslash) \Longleftrightarrow A e=e \text { and } \tilde{\mathcal{A}} z(\oslash)=e \Longleftrightarrow A e=e
$$

Ilence, the preconsistency conditions sum up to $A e=e$. In order to present the order conditions, we define as in [1] :

$$
C_{0}=A e-e \text { and } C_{j}=A(c-e)^{j}+j\left[B(c-e)^{j-1}+D c^{j-1}\right]-c^{j}, j=1,2, \cdots
$$

Theorem 1 A zero-stable and preconsistent $k$-dimensional block method is of order $p \geq 1$ iff:

$$
\begin{array}{r}
\forall j, 0 \leq j \leq p-1, C_{j}=0 \\
\mathbb{E}\left(C_{\mathfrak{p}}, 0\right)^{T}=(0,0)^{T}
\end{array}
$$




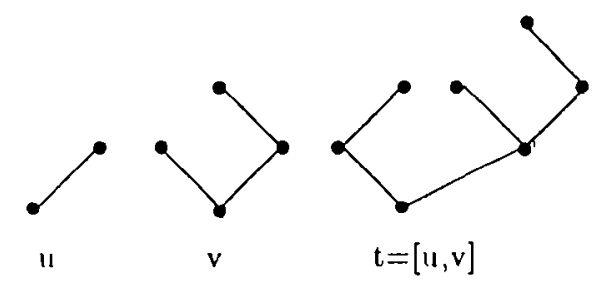

Figure 2: Recursive definition of trees

proof : we first recall that the local crror of a general linear method is given by

$$
d_{i}^{n+1}=z_{i}\left(x_{n}+h, h\right)-\sum_{j=1}^{2 k} \mathcal{A}_{i, j} z_{j}\left(x_{n}, h\right)-\sum_{j=1}^{k} \mathcal{B}_{i, j} h F\left(v_{j}\right)
$$

where

$$
v_{i}=\sum_{j=1}^{2 k} \tilde{\mathcal{A}}_{i, j} z_{j}\left(x_{n}, h\right)+\sum_{j=1}^{k} \tilde{\mathcal{B}}_{i, j} h F\left(v_{j}\right)
$$

By writing $v_{i}$ and $d_{i}^{n+1}$ as Butcher-series, we get from (4) and (5) :

$$
\begin{array}{r}
d_{i}(t)=\left(p z_{i}\right)(t)-\sum_{j=1}^{2 k} \mathcal{A}_{i, j} z_{j}(t)-\sum_{j=1}^{k} \mathcal{B}_{i, j} v_{j}^{\prime}(t) \\
v_{i}(t)=\sum_{j=1}^{2 k} \tilde{\mathcal{A}}_{i, j} z_{j}(t)+\sum_{j=1}^{k} \tilde{\mathcal{B}}_{i, j} v_{j}^{\prime}(t)
\end{array}
$$

where

$$
\left(p z_{i}\right)(t)=\sum_{j=0}^{\rho(t)}\left(\begin{array}{l}
\rho(t) \\
j
\end{array}\right) z_{i}\left(\tau^{j}\right)
$$

(see [1] pp. 398). A necessary and sufficient condition for a general linear method to be of order $p$ is given by the theorem 8.14 in [1] :

$$
\begin{array}{r}
\forall t \in T, \rho(t) \leq p-1, d(t)=0 \\
\forall t \in T, \rho(t)=p, \mathbb{E} d(t)=0
\end{array}
$$

Let us show that under assumption (6) we have the following property (denoted by $P_{p-1}$ ) :

$$
\forall t \in T, \rho(t) \leq p-1, v(t)=v\left(\tau^{\rho(t)}\right)=c^{\rho(t)}=A(c-e)^{\rho(t)}+\rho(t)\left[B(c-e)^{(\rho(t)-1)}+D c^{\rho(t)}\right]
$$

If we assume that $P_{q-1}$ is valid for $q-1 \leq p-2$, then we may compute explicitely $v(t)$ and $d(t)$ for any tree of order $q$ :

$$
v^{\prime}(t)=\rho(t) v\left(t_{1}\right) \cdots v\left(t_{m}\right)
$$

where $t=\left[t_{1}, \cdots, t_{n}\right]$ denotes the tree which leaves over the trees $t_{i}$ when its root and the adjaccnt branches are chopped off (see fig. 2). From $\rho\left(t_{i}\right) \leq q-1$ and the hypothesis $\left(P_{q-1}\right)$ we get :

$$
v\left(t_{i}\right)=c^{\rho\left(t_{1}\right)}
$$


This identity implies $v^{\prime}(t)=\rho(t) . c^{\left(\sum_{s=1}^{m} \rho\left(t_{i}\right)\right)}=q \cdot c^{g-1}$. If we then insert $v^{\prime}(t)$ into $v(t)$ we get :

$$
v(t)=\dot{A} z\left(\tau^{q}\right)+\rho(t) \tilde{B} c^{q-1}
$$

This expression can be used to compute $d(t)$ as follows :

$$
\begin{aligned}
d(t) & =(p z)(l)-\mathcal{A} z\left(\tau^{q}\right)-\mathcal{B} v^{\prime}\left(\tau^{q}\right) \\
& =\left(\begin{array}{c}
\sum_{j=0}^{q}\left(\begin{array}{l}
q \\
j
\end{array}\right)(c-e)^{j} \\
\sum_{j=0}^{q}\left(\begin{array}{l}
q \\
j
\end{array}\right) j \cdot(c-e)^{j-1}
\end{array}\right)-\left(\begin{array}{cc}
A & B \\
0 & 0
\end{array}\right)\left(\begin{array}{c}
(c-e)^{q} \\
q \cdot(c-e)^{(q-1)}
\end{array}\right)-\left(\begin{array}{c}
D \\
I
\end{array}\right) q \cdot c^{(q-1)} \\
& =\left(\begin{array}{c}
c^{q}-\left\{A(c-e)^{q}+q \cdot\left[B(c-e)^{(q-1)}+D c^{(q-1)}\right]\right\} \\
0
\end{array}\right)
\end{aligned}
$$

Since $d(t)=0$, we finally have for any tree of order $q$ :

$$
v(t)=v\left(\tau^{q}\right)=c^{q}=A(c-e)^{q}+q \cdot\left[B(\dot{c}-e)^{(q-1)}+D c^{(q-1)}\right]
$$

We know that the method is preconsistent and that $\tau$ is the only tree of order one, so that $P_{1}$ is true. Hence, an induction argument allows us to conclude that $P_{p-1}$ is also valid. Similarly, we can get for any tree of order $p, d(t)=c^{p}-\left\{A(c-e)^{p}+p \cdot\left[B(c-e)^{(p-1)}+D c^{(p-1)}\right]\right\}$. This leads to:

$$
\mathbb{E} d(t)=0 \Longleftrightarrow \mathbb{E}\left(\begin{array}{c}
C^{p} \\
0
\end{array}\right)=\left(\begin{array}{l}
0 \\
0
\end{array}\right)
$$

QED.

Corollary $1 A$ sufficient condition for a $k$-dimensional block method to be of order $p \geq 1$ is given by :

$$
\forall j \in[0, p], C_{j}=0
$$

Remark 1 This result has been obtained by B.P. Sommeijer, W. Couzy and P.J. van der Houwen in [4] by using a componentwise definition of the order.

In order to completely define the order conditions, we need to compute matrix $\mathbb{E}$. However, its decomposition as $T \cdot \operatorname{diag}(I, 0, \cdots, 0) \cdot T^{-1}$ is not of practical interest, as far as we aim at deriving purely formal algebraic conditions. The following theorem together with the next lemma give us an easy way to compute $\mathbb{E}$ from the characteristic polynomial of $A$.

Theorem 2 Let us assume that the method is stable and preconsistent. Let l be the multiplicity of the eigenvalue 1 of $\mathcal{A}$, and let $Q$ be the polynomial defined by :

$$
(x-1)^{\prime} Q(x)=\operatorname{det}(\mathcal{A}-x \mathcal{I})
$$

We may then write $\mathbb{E}$ as :

$$
\mathbb{E}=\frac{Q(\mathcal{A})}{Q(1)}
$$

proof: Let $T=\left[T_{1}, T_{2}\right]$ be the decomposition of $T$ onto $T_{1}$ the basis of the eigenspace associated with 1 and $T_{2}$ the basis of the eigenspace associated with others eigenvalues. Then we have:

$$
\mathcal{A}=T\left(\begin{array}{cc}
I & 0 \\
0 & \mathcal{R}
\end{array}\right) T^{-1}
$$


Therefore, $\operatorname{det}(x \cdot \mathcal{I}-\mathcal{A})=(x-1)^{\prime} \cdot \operatorname{det}(x \cdot I-\mathcal{R})$. It promptly derives that :

$$
Q(\mathcal{A})=T\left(\begin{array}{cc}
Q(I) & 0 \\
0 & Q(\mathcal{R})
\end{array}\right) T^{-1}
$$

Since $Q$ is the characteristic polynomial of $\mathcal{R}$ (or its opposite) we have $Q(\mathcal{R})=0$. Hence, it follows that :

$$
\mathbb{E}=\frac{Q(\mathcal{A})}{Q(1)}
$$

Lemma 1 Under the hypothesis of previous theorem, $\mathbb{E}$ may be writlen as :

$$
\mathbb{E}=\left(\begin{array}{cc}
\frac{Q(\boldsymbol{A})}{Q(1)} & \Delta \\
0 & \frac{Q(0)}{Q(1)} I
\end{array}\right)
$$

where $\Delta \in \mathbb{R}^{k \times k}$

proof : let $\lambda_{1}, \lambda_{2}$ be two roots of $Q$. We have :

$$
\mathcal{A}-\lambda_{i} \mathcal{I}=\left(\begin{array}{cc}
A-\lambda_{i} I & \Delta_{i} \\
0 & \left(-\lambda_{i}\right) I
\end{array}\right) \text { with } \Delta_{i}=B \text { for } i=1,2
$$

Hence, $\left(\mathcal{A}-\lambda_{1} \mathcal{I}\right)\left(\mathcal{A}-\lambda_{2} \mathcal{I}\right)$ may be written as :

$$
\left(\begin{array}{cc}
\left(A-\lambda_{1} l\right)\left(A-\lambda_{2} I\right) & A B-\left(\lambda_{1}+\lambda_{2}\right) B \\
0 & \left(-\lambda_{1}\right)\left(-\lambda_{2}\right) I
\end{array}\right)
$$

which is of the desired type if we define $\Delta=A B-\left(\lambda_{1}+\lambda_{2}\right) B$. By considering the decomposition of $Q$ on $\mathbb{C}$, we can conclude similarly for $\mathbb{E}$.

\subsection{Stability}

Definition $5 \mathrm{~A} k$-dimensional block method is called zero-stable if it exists a constant $K$ such that :

$$
\forall n \in \mathbb{N},\left\|A^{n}\right\| \leq K
$$

Remark 2 By considering the form of $S=\mathcal{A}$, it is easily seen that this definition is cquivalent to those of the previous section.

The linear stability of block methods can be investigated by applying the method to the test equation $y^{\prime}=\lambda y$. This leads to a rccursion of the form :

$$
Y_{n+1}=M(z) Y_{n}
$$

where $M(z):=(I-z . D)^{-1}(A+z . B)$ is called the amplification matrix and where $z=\lambda h$ is defined as usual. Following the familiar notions of stability, we will define successively :

Definition 6 The stability region of a block method is the region of the complex plane where the sequence of vectors $\left(Y_{n}\right)_{n \in \mathbb{N}}$ remains bounded for any initial vector $Y_{0}$.

Definition $7 \mathrm{~A} k$-dimensional block method is called $A$-stable if the stability region contains the left half plane $\mathbb{C}^{-}=\{z \in \mathbb{C} ; \operatorname{Re}(z) \leq 0\}$. 
Definition 8 A $k$-dimensional block method is called L-stable if it is A-stable and if it has an am. plification matrix with vanishing eigenvalues at infinity.

By expressing $M(z)=\left(\frac{I}{z}-D\right)^{-1}\left(B+\frac{A}{z}\right)$, we obtain :

$$
\lim _{|z| \rightarrow \infty} M(z)=-D^{-1} B
$$

Therefore, the amplification matrix of an L-stable matrix is such that $\rho\left(D^{-1} B\right)=0$.

Here we observe that a point $z$ belongs to the stability region if and only if the eigenvalues of the amplification matrix $M(z)$ have a modulus less or equal to one and the eigenvalues of modulus one are non-defective. However, it seems difficult to investigate the stability region from this single characterization. A more appropriate result establishes sufficient conditions for A-stability. This will enable us to "verify" that a method is A-stable.

Lemma 2 Let a $k$-dimensional block method be defined by the matrices $A, B$ and $D \in \mathbb{R}^{k \times k}$. Let $P(x, z)=\operatorname{det}(M(z)-x . I)$ denote the characteristic polynomial of $M(z)$, and let $\alpha_{i}(z), i=1, \cdots, 2 k$ be the coefficients of $(w-1)^{2 k} P\left(\frac{w+1}{w-1}, z\right) \vec{P}\left(\frac{w+1}{w-1}, z\right)$. Finally, let the matrix $\mathcal{H}$ be :

$$
\mathcal{H}(z)=\left(\begin{array}{cccccc}
\alpha_{2 k-1} & \alpha_{2 k-3} & \alpha_{2 k-5} & \cdots & \cdots & \alpha_{-2 k+1} \\
\alpha_{2 k} & \alpha_{2 k-2} & \alpha_{2 k-4} & \cdots & \cdots & \alpha_{-2 k+2} \\
0 & \alpha_{2 k-1} & \alpha_{2 k-3} & \cdots & \cdots & \alpha_{-2 k+3} \\
0 & \alpha_{2 k} & \alpha_{2 k-2} & \cdots & \cdots & \alpha_{-2 k+4} \\
\vdots & \vdots & \vdots & & & \vdots \\
0 & 0 & 0 & \cdots & \cdots & \alpha_{0}
\end{array}\right)
$$

Then a sufficient condition for the method to be A-stable is given by:

$$
\forall l \in[1,2 k], \forall y \in \mathbb{R}^{*}, \operatorname{det}\left(\left(h_{i j}(i y)\right)_{1 \leq i, j \leq l)}>0\right.
$$

proof : since the spectral radius of $M(z)$ satisfies the maximum principle, the method is A-stable if and only if it is stable on the imaginary axis and $D$ has strictly positive coefficients. In order to work with a real polynomial, we substitute $P \bar{P}$ to $P$. For $z=i y,\left(i^{2}=-1\right)$ we have :

$$
P(x, i y) \bar{P}(x, i y)=\left(x^{k}+\sum_{n=0}^{k-1} p_{n}(i y) x^{n}\right)\left(x^{k}+\sum_{n=0}^{k-1} \overline{p_{n}(i y)} x^{n}\right)
$$

Now, we know that the roots of $P \bar{P}$ are those of $P$ together with their conjugate. By applying the mapping $x=\frac{w+1}{w-1}$ to the unit disc, which is a one-to-one function between the open unit disc $|x|<1$ and the left half plane $R e w<0$, we obtain the following condition :

$$
\forall y \in \mathbb{R}^{*}, W(w):=(w-1)^{2 k} P\left(\frac{w+1}{w-1}, i y\right) \bar{P}\left(\frac{w+1}{w-1}, i y\right)=0 \Longrightarrow \operatorname{Re}(w)<0
$$

Now, the result is simply a consequence of the Routh-Hurwitz criterium, applied to the polynom $W \square$

\section{A Two-Dimensional L-Stable third-order method}

In this section we look for $L$-stable methods of maximum order when $k=2$. The preconsistency condition gives :

$$
a_{11}+a_{12}=1 \text { and } a_{21}+a_{22}=1
$$


The free coefficients of the method sum up to $a_{11}$ and $a_{21} . A$ has the following expression:

$$
A=\left(\begin{array}{ll}
a_{1} & 1-a_{1} \\
a_{2} & 1-a_{2}
\end{array}\right)
$$

To get zero-stability, we must have the following condition:

$$
-1 \leq a_{1}-a_{2} \leq 1
$$

Therefore, the eigenvalues of $\mathcal{A}$ are $1, a_{1}-a_{2}$ and 0 . The expression of $\mathcal{A}$ shows that the eigenspace associated with 0 is of dimension 2 at least. 0 is consequently a root of multiplicity one of the minimal polynomial of $\mathcal{A}$ or of multiplicity two if $a_{1}-a_{2}=0$. Hence, we can take $Q(x)=x\left[x-\left(a_{1}-a_{2}\right)\right]$ and compute matrix $\mathbb{E}$ :

$$
Q(1)=1-a_{1}+a_{2}
$$

and :

$$
Q(A)=A\left(A-\left(a_{1}-a_{2}\right) I\right)=\left(\begin{array}{ll}
a_{2} & 1-a_{1} \\
a_{2} & 1-a_{1}
\end{array}\right)
$$

- Corollary 2 Let $k=2$ and let us suppose that the method is zero-stable and preconsistent. Then, it is of order $p \geq 1$ iff :

$$
\begin{array}{r}
\forall j, 0 \leq j \leq p-1, C_{j}=0 \\
\Pi C_{p}=0
\end{array}
$$

with either :

if $a_{2} \neq 0$ or $a_{1} \neq 1$, or $\Pi=I$ else.

$$
\Pi=\left(\begin{array}{ll}
a_{2} & 1-a_{1} \\
a_{2} & 1-a_{1}
\end{array}\right)
$$

By choosing appropriate coefficients, one can find a L-stable method of order 3. An elementary calculation shows that $C_{i}, i=0,1,2$ vanish if :

$$
b_{j 1}=\frac{1}{2}(1-c) a_{j}+\frac{c_{j}\left(2 d_{j}-c_{j}\right)}{2(1-c)}, b_{j 2}=c_{j}+(1-c) a_{j}-b_{j 1}-d_{j}, c_{1}=c, c_{2}=1, \text { for } j=1,2
$$

In addition to this, $\Pi C_{3}=0$ implies :

$$
\begin{aligned}
& 3 a_{2} a_{1} c^{3}+9 a_{2} a_{1} c-3 a_{2} a_{1}+6 a_{2} b_{11} c-6 a_{2} b_{11}-3 a_{2} c^{3}-9 a_{1} a_{2} c^{2}+6 a_{1} b_{21} c^{2}-18 a_{1} b_{21} c \\
+ & 12 a_{1} b_{21}+a_{1}+9 a_{2} c^{2}-12 a_{2} c+5 a_{2}-6 b_{21} c^{2}+18 b_{21} c-12 b_{21}-1=0
\end{aligned}
$$

as far as we assume that $a_{1} \neq 1$ or $a_{2} \neq 0$. If we further impose that $M(\infty)$ has null eigenvalues, we get :

$$
\begin{aligned}
b_{11} b_{22}-b_{12} b_{21} & =0 \\
d_{1} b_{22}+d_{2} b_{11} & =0
\end{aligned}
$$

Solving the previous relations and stability constraints now gives :

$$
\begin{aligned}
& A=\left(\begin{array}{cc}
3 \frac{-147-1880 b_{21}+2000 b_{21}^{2}}{2800 b_{21}^{2}-2760 b_{21}-49} & -8 \frac{400 b_{21}^{2}-360 b_{21}-49}{2800 b_{21}^{2-27600 b_{21}-49}} \\
9 / 5 & -4 / 5
\end{array}\right), \\
& B=\left(\begin{array}{cc}
-120 \frac{b_{21}\left(7+20 b_{21}\right)}{2800 b_{21}^{2}-2760 b_{21}-49} & 3 \frac{49+280 b_{21}+400 b_{21}^{2}}{2800 b_{21}^{2}-2760 b_{21}^{2}-49} \\
b_{21} & -7 / 40-1 / 2 b_{21}
\end{array}\right), \\
& D=\left(\begin{array}{cc}
120 \frac{b_{21}\left(-11+20 b_{21}\right)}{2800 b_{21}^{2}-2760 b_{21}-49} & 0 \\
0 & 11 / 40-1 / 2 b_{21}
\end{array}\right)
\end{aligned}
$$


where $c=3 / 2$ and $b_{21}=\operatorname{Root} O f\left(4361-36180 Z+158800 Z^{2}+18000 Z^{3}\right) \approx-3.5308655705748385794$.

We sliall now study the stability on the imaginary axis. The condition of lemma (2) leads to the following calculations :

$$
P(x, z)=x^{2}+p_{1}(z) x+p_{0}(z)
$$

with :

$$
\begin{aligned}
& p_{1}(z)=\frac{z d_{1}-a_{1}+a_{2}-1-z b_{11}+z^{2} b_{11} d_{2}+z^{2} d_{1} b_{22}-z b_{22}-z d_{1} a_{2}+a_{1} z d_{2}}{\left(z d_{1}-1\right)\left(z d_{2}-1\right)} \\
& p_{0}(z)=\frac{a_{1} z b_{22}-z^{2} b_{12} b_{21}+a_{1} z b_{21}-z b_{11} a_{2}+z^{2} b_{11} b_{22}-z b_{12} a_{2}-a_{2}+a_{1}+z b_{11}-z b_{21}}{\left(z d_{1}-1\right)\left(z d_{2}-1\right)}
\end{aligned}
$$

so that

$$
\begin{aligned}
P(x, i y) F(x, i y) & =\left(x^{2}+p_{1}(i y) x+p_{0}(i y)\right)\left(x^{2}+\overline{p_{1}(i y)} x+\overline{p_{0}(i y)}\right) \\
& =x^{4}+\left(p_{1}+\overline{p_{1}}\right) x^{3}+\left(p_{0}+\overline{p_{0}}+\left|p_{1}\right|^{2}\right) x^{2}+\left(\overline{p_{1}} \overline{p_{0}}+p_{0} \overline{p_{1}}\right) x+\left|p_{0}\right|^{2}
\end{aligned}
$$

The leading cocficient $\left|1+p_{1}+p_{0}\right|$ of $W$ is :

$-\frac{z\left(b_{21} d_{1}^{2} a_{2}-b_{21} a_{1} d_{2} d_{1}-b_{11} d_{2} b_{21}-a_{1} b_{21}^{2} d_{1}+b_{11} a_{2} d_{1} b_{21}+a_{1} b_{11} d_{2} b_{21}-b_{11}^{2} d_{2} a_{2}+b_{21}^{2} d_{1}-d_{1}^{2} b_{21} z d_{2}+d_{1} b_{21} d_{2}\right)}{d_{1} b_{21}\left(z d_{1}-1\right)\left(z d_{2}-1\right)}$

so that it is non-zero for all $z=i . y \neq 0$. Rather than working with $W$, we prefer to work with $W /\left|1+p_{1}+p_{0}\right|^{2}$. The coefficients of $W(w, i . y) /\left|1+p_{1}(i . y)+p_{0}(i . y)\right|^{2}=w^{4}+\alpha(y) w^{3}+\beta(y) w^{3}+$ $\gamma(y) w+\delta(y)$ have the following expression :

$$
\begin{aligned}
& \alpha(y)=\frac{-2\left(p_{1}(i . y) \overline{p_{0}(i . y)}+p_{0}(i . y) \overline{p_{1}(i . y)}\right)-4\left|p_{0}(i . y)\right|^{2}+4+2\left(p_{1}(i . y)+\overline{p_{1}(i . y)}\right)}{\left|1+p_{1}(i . y)+p_{0}(i . y)\right|^{2}} \\
& \beta(y)=\frac{6\left|p_{0}(i . y)\right|^{2}+6-2\left|p_{1}(i . y)\right|^{2}-2\left(p_{0}(i . y)+\overline{p_{0}(i . y)}\right)}{\left|1+p_{1}(i . y)+p_{0}(i . y)\right|^{2}} \\
& \gamma(y)=\frac{4-4\left|p_{0}(i . y)\right|^{2}-2\left(p_{1}(i . y)+\overline{p_{1}(i . y)}\right)+2\left(p_{0}(i . y) \overline{p_{1}(i . y)}+p_{1}(i . y) \overline{p_{0}(i . y)}\right)}{\left|1+p_{1}(i . y)+p_{0}(i . y)\right|^{2}} \\
& \delta(y)=\frac{\left|p_{1}(i . y)-p_{0}(i . y)-1\right|^{2}}{\left|1+p_{1}(i . y)+p_{0}(i . y)\right|^{2}}
\end{aligned}
$$

So that the Routh-Hurwitz conditions are

$$
\forall y \in \mathbb{R}^{*}, \alpha(y)>0, \alpha(y) \beta(y)-\gamma(y)>0,(\alpha(y) \beta(y)-\gamma(y)) \gamma(y)-\alpha^{2}(y) \delta(y)>0, \delta(y)>0
$$

It is then possible to verify that $(11)$ is satisfied for any $y \in \mathbf{R}^{*}$ by using symbolic calculus. Since $d_{1}$ and $d_{2}$ are positive, lemma (2) shows that the method is A-stable. Furthermore, the eigenvalues of $M(\infty)$ are null by construction.

\section{Generalized backward differentiation formulas}

It soon becomes very tedious to compute the order conditions and to check A-stability or even stability on the real axis. A way to overcome these obstacles is to perform a numerical search in the space of free coefficients, once the conditions for zero-stability and order $p$ have been derived. This technique has been investigated by B.P. Sommeijer, W. Couzy and P.J. van der Houwen in [4]. They have reached the fifth order with almost A-stability. However, no formal proof of these results was 
given. In addition to this, it seems hard to go further owing to the considerable amount of work needed to find optimal coefficients. This is why we have turned to the more simple case where $B$ is nult, even if it dramatically reduces the number of free coefficients : for a given block-size $k$ and a given vector $c \in \mathbb{R}^{k}$, there are $(k+1) k$ unknowns coefficients in $A$ and $D$ instead of $(2 k+1) k$. Since every order condition $C_{j}=0$ corresponds to $k$ linear equations, we do not expect more than $k^{\text {th }}$-order methods.

\subsection{Construction}

Let us define the following $k \times k$ matrices :

$$
U=\left(\begin{array}{ccccc}
1 & c_{1}-1 & \left(c_{1}-1\right)^{2} & \cdots & \left(c_{1}-1\right)^{k-1} \\
1 & c_{2}-1 & \left(c_{2}-1\right)^{2} & \cdots & \left(c_{2}-1\right)^{k-1} \\
\vdots & \vdots & & \vdots & \vdots \\
1 & c_{k-1}-1 & \left(c_{k-1}-1\right)^{2} & \cdots & \left(c_{k-1}-1\right)^{k-1} \\
1 & c_{k}-1 & \left(c_{k}-1\right)^{2} & \cdots & \left(c_{k}-1\right)^{k-1}
\end{array}\right)
$$

and

$$
V=\left(\begin{array}{ccccc}
1 & -d_{1}+c_{1} & -2 d_{1} c_{1}+c_{1}^{2} & \cdots & -(k-1) d_{1} c_{1}^{k-2}+c_{1}^{k-1} \\
1 & -d_{2}+c_{2} & -2 d_{2} c_{2}+c_{2}^{2} & \cdots & -(k-1) d_{2} c_{2}^{k-2}+c_{2}^{k-1} \\
\vdots & \vdots & & \vdots & \vdots \\
1 & -d_{k-1}+c_{k-1} & -2 d_{k-1} c_{k-1}+c_{k-1}^{2} & \cdots & -(k-1) d_{k-1} c_{k-1}^{k-2}+c_{k-1}^{k-1} \\
1 & -d_{k}+c_{k} & -2 d_{k} c_{k}+c_{k}^{2} & \cdots & -(k-1) d_{k} c_{k}^{k-2}+c_{k}^{k-1}
\end{array}\right)
$$

where $\left(d_{i}\right)_{1 \leq i \leq k}$ are the diagonal elements of $D$. For a given diagonal matrix $D$, the set of conditions $C_{j}=0, j=0, \cdots, k-1$ can be expressed by the matrix relation $A . U=V$. Since $U$ is a non-singular Vandermonde matrix (the $c_{i}$ 's are assumed to be pairwise distinct), $A$ is entirely determined and the resulting method is at least of order $k-1$.

Definition 9 We call $\mathcal{M}(k)$ a $k$-dimensional parallel block method where:

- $c=(1,2, \cdots, k)^{T} \in \mathbb{R}^{k}$

- $B=0$

- D has the following expression:

$$
D=\frac{1}{r}\left(\begin{array}{ccccc}
c_{1} & 0 & \cdots & \cdots & 0 \\
0 & c_{2} & 0 & \cdots & 0 \\
\vdots & \ddots & \ddots & \ddots & \vdots \\
0 & \cdots & \cdots & \cdots & c_{k}
\end{array}\right)+\frac{1}{r} I, r \in \mathbb{R}^{*}
$$

- $A=V \cdot U^{-1}$

\subsection{Zero-stability and stability at infinity}

Theorem 3 Let us assume that $r \geq \frac{p}{2}$. Then the method $\mathcal{M}(k)$ satisfies :

- $\mathcal{M}(k)$ is of order at least $k-1$.

- $\mathcal{M}(k)$ is zero-stable.

- $\mathcal{M}(k)$ is stable in the left-half plane $x \leq \frac{1}{r} \min _{1 \leq i \leq k} c_{i}-\left\|D^{-1} A\right\|$. 
proof : by definition of $A$, the method is of order $p=k-1$. Since $\left(c_{i}\right)_{1 \leq i \leq k}$ are pairwise distinct $e, c, \cdots, c^{k-1}$ is a basis of $\mathbb{R}^{k}$ where matrix $A$ has a reduced form. According to the order conditions of Corollary 1 we indeed have:

$$
A e=e \text { and } \forall n \in[1, \ldots, p], A c^{n}=\left(1-\frac{n}{r}\right) c^{n}-\frac{n}{r} c^{n-1}-\sum_{j=0}^{n-1} C_{j}^{n}(-1)^{n-j} A c^{j}
$$

Hence, an easy induction argument shows that $A$ is upper-triangular. We may observe that $D$ is has also a very simple expression in this basis. As a matter of fact, we have :

$$
\forall n \in[0, p], D c^{n}=\frac{1}{r}\left(c^{n+1}+c^{n}\right)
$$

so that :

$$
D=\frac{1}{r}\left(\begin{array}{cccccc}
1 & 0 & \cdots & \cdots & 0 & \alpha_{1} \\
1 & 1 & 0 & \cdots & 0 & \alpha_{2} \\
0 & \ddots & \ddots & \ddots & & \vdots \\
\vdots & & \ddots & \ddots & \ddots & \\
0 & \cdots & \cdots & 0 & 1 & \alpha_{k-1} \\
0 & \cdots & \cdots & 0 & 1 & 1+\alpha_{k}
\end{array}\right)
$$

where $\left(\alpha_{i}\right)_{1 \leq i \leq k}$ are the components of $c^{k}$ in basis $\left\{e, c, \cdots, c^{k-1}\right\}$. From the expression of $A$ we can see that the eigenvalues of $A$ are $1-j / r$ for $j=0, \cdots, p$. They are pairwise distinct and of modulus less or equal to one, so that $\mathcal{M}(k)$ is zero-stable. Finally, by considering the Laplace transform $\mathcal{L} f$ of $f(t)=e^{-t D^{-1}} D^{-1} A$ we get :

$$
\begin{aligned}
\mathcal{L} f(-z) & =\int_{0}^{\infty} e^{-t D^{-1}} D^{-1} A e^{z . t} d t \\
& =\int_{0}^{\infty} e^{-t\left(D^{-1}-z I\right)} D^{-1} A d t \\
& =(I-z \cdot D)^{-1} A \\
& =M(z)
\end{aligned}
$$

It follows that :

$$
\begin{aligned}
\|M(z)\| & \leq\left\|D^{-1} A\right\| \int_{0}^{\infty} e^{-\left(x+\frac{\varepsilon}{r}\right) t} d t \\
& \leq \frac{\left\|D^{-1} A\right\|}{x+c / r}
\end{aligned}
$$

where $c=\min _{1 \leq i \leq k} c_{i}$ et $z=-x+i y$.

\subsection{L-stability}

The estimation of the previous theorem shows that when $\operatorname{Re}(z)$ tends to infinity, $\|M(z)\|$ tends to zero. In fact, this result is also valid when $|z|$ tends to infinity, since $\rho\left(D^{-1} B\right)=0$ (see section 2.6). It follows that L-stability is implied by A-stability for methods $\mathcal{M}(k)$. Nevertheless, A-stability can not be easily obtained, and we actually did not find any direct proof. All we can show is indeed that methods $\mathcal{M}(k)$ satisfy the conditions of lemma 2 for certain values of $r$ with respect to $k$. This can be done by using an efficient symbolic calculus system. 


\begin{tabular}{|c|c|c|c|}
\hline \hline Block size & Order & $\mathrm{r}$ & Error Constant \\
\hline 2 & 2 & 4 & $(1 / 2,1 / 6)^{T}$ \\
\hline 3 & 2 & $11 / 2$ & $(-1 / 6,1 / 3,11 / 24)^{T}$ \\
\hline 4 & 4 & 5 & $(1 / 12,-1 / 12,1 / 4,13 / 12)^{T}$ \\
\hline 5 & 4 & 6 & $(-1 / 20,1 / 30,-1 / 20,1 / 5,77 / 60)^{T}$ \\
\hline 6 & 5 & 6 & $(1 / 30,-1 / 60,1 / 60,-1 / 30,1 / 6,223 / 140)^{T}$ \\
\hline 7 & 6 & 6 & $(-1 / 42,1 / 105,-1 / 140,1 / 105,-1 / 42,1 / 7,481 / 280)^{T}$ \\
\hline 8 & 7 & 7 & $(1 / 56,-1 / 168,1 / 280,-1 / 280,1 / 168,-1 / 56,1 / 8,4889 / 2520)^{T}$ \\
\hline
\end{tabular}

Table 1: Normalized error vectors and values of $\mathbf{r}$

Remark 3 Parameter $r$ is first estimated numerically and then rounded to an integer. When more than one choice was possible, we tried to "minimize" the error constant.

We present in appendix $\mathrm{H}$ a program written in Maple which purpose is to build methods $\mathcal{M}(k)$ for any value of $k$ and $r$. This program then assembles matrix $\mathcal{H}$ and computes all its minors, which are known to be polynomials of real variable $y$. By definition of $\mathcal{H}$ and of the resulting polynomials, it is known that only even exponents of $y$ appear, so that an easy criterium to check for A-stability is to verify that all coefficients in $\operatorname{det}\left(\mathcal{H}_{i, j}\right)_{1 \leq i, j \leq l, l}=1, \cdots, 2 k$ are positive. This has been done up to $k=8$. Higher values of $k$ would require more than a week of computations on a SPARC station 2. That is why such values of $k$ have not been investigated. The program returns 1 if A-stability is achieved, whereas 0 means that the criterium used is not satisfied.

Remark 4 For higher values of $k$ we can investigate the stability on the real axis in a similar manner (here we have to compute the matrix $\mathcal{H}$ directly from $P$, instead of $P \bar{P}$ ). This work has been carried out with $r=k-1$ up to $k=12$, showing that $\mathcal{M}(k)$ is $A_{0}$-stable at least up to order 11 .

As in [4] we introduce the normalized error vectors in order to compare their components with the error constants corresponding to conventional linear multistep methods :

$$
E_{j}:=\frac{C_{j}}{j ! D e}
$$

where the division of vectors is meant componentwise. We now conclude with a survey of the results obtained up to $k=8$ and we give the root locus curves and the matrices $A$ and $D$ for the different methods in appendix from $A$ to $G$. It should be noticed that for $k=2$ and $k=4$ the order of the method is $k$ instead of $k-1$ as stated in Theorem 3. As a matter of fact, we have chosen $r$ such that $\mathbb{E} C_{k}=0$. As seen in Theorem 1, this additional condition is sufficient for order $k$. A close look at condition $\mathbb{E} C_{k}=0$ shows that there exists a solution $r$ for $k=1, \cdots, 8$. However, it is generally not integer and not even a rational, so that the resulting method has no longer nice rational coefficients. As mentionned above, for $k>8$ no result has been obtained, owing to the amount of computations needed. An alternative approach is however possible, provided we consider a combined local-numerical analysis is sufficient. Though it does not seem possible to conclude on the L-stability of $\mathcal{M}(k), k>8$ only from the graph of $\rho(M(z)$ on the imaginary axis (see for instance fig. 3), we may observe that outside a neighborhood of the origin, $\rho(M(i . y)$ is less than one. In this neighborhood, we may use the following result :

Lemma 3 Let us assume that $\mathcal{M}(k)$ is a zero-stable method of order $p$ with $r>p / 2$ and let $\lambda(z)$ be the continuation of the eigenvalue 1 of $A$. If we denote by $1-K_{1} \cdot z^{p+1}+K_{2} \cdot z^{p+2}+O\left(z^{p+3}\right)$ the truncation of series $e^{-z} \cdot \lambda(z)$, then $\mathcal{M}(k)$ is strongly zero-stable if one of the following two cases occurs:

- $\exists q \in \mathbb{N}, p=2 q$ and $(-1)^{q} . K_{2}>0$ 


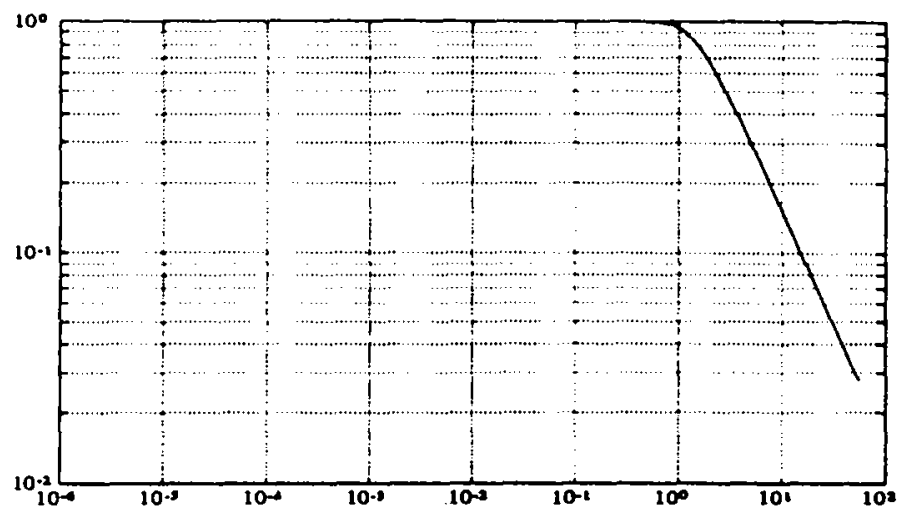

Figure 3: $\rho(M(i . y))$ in $\log -\log$ scale for $\mathcal{M}(9)$ with $r=8$

- $\exists q \in \mathbb{N}, p=2 q+1$ and $(-1)^{q+1} \cdot K_{1}>0$

proof : since $r>p / 2$, we know that all eigenvalues of $A$ have a modulus strictly less than 1 except for the one-fold eigenvalue 1 , so that there exists a neigborhood of the origin, in the complex plane, in which these eigenvalues are bounded in modulus by 1 (by continuity of the roots). As for eigenvalue 1, we may write it as a truncated Taylor series since it is a simple root of the characteristic polynomial of $A$. Since $\mathcal{M}(k)$ is of order $p$, we have :

$$
e^{-3} \cdot \lambda(z)=1-K_{1} \cdot z^{p+1}+K_{2} \cdot z^{p+2}+O\left(z^{p+3}\right)
$$

Let $z=i y$. For $p=2 q+1$ we have:

$$
\left|e^{-3} \cdot \lambda(z)\right|^{2}=1-2(-1)^{q+1} K_{1} y^{2 q+2}+K_{1}^{2} y^{4 q+4}+K_{2}^{2} y^{4 q+6}+O\left(y^{2 q+4}\right)
$$

so that, $\left|e^{-z} \cdot \lambda(z)\right| \leq 1$ in a neighborhood of 0 if and only if $(-1)^{q} \cdot K_{1}>0$.

As for $p=2 q$ :

$$
\left|e^{-z} \cdot \lambda(z)\right|^{2}=1+2(-1)^{q+1} K_{2} y^{2 q+2}+K_{1}^{2} y^{4 q+2}+K_{2}^{2} y^{4 q+4}+O\left(y^{2 q+3}\right)
$$

so that, $\left|e^{-z} \cdot \lambda(z)\right| \leq 1$ if and only if $(-1)^{4} \cdot K_{2}>0$.

By computing Taylor series of eigenvalue 1 , one can prove that Methods $\mathcal{M}(k), k=9,10$ with respectively $r=8,9$ satisfy the conditions of previous lemma. This result together with stability plots are strong presumptions for their L-stability. 


\begin{tabular}{|c|c|c|c|c|c|c|c|}
\hline $\mathrm{k}$ & $\mathrm{h}=1 / 4$ & $\mathrm{~h}=1 / 8$ & $\mathrm{~h}=1 / 16$ & $\mathrm{~h}=1 / 32$ & $\mathrm{~h}=1 / 64$ & $\mathrm{~h}=1 / 128$ & $\mathrm{~h}=1 / 256$ \\
\hline 2 & 2.57 & 3.13 & 3.75 & 4.37 & 4.98 & 5.58 & 6.18 \\
\hline 3 & 2.63 & 3.23 & 3.83 & 4.43 & 5.03 & 5.64 & 6.24 \\
\hline 4 & 3.75 & 4.74 & 5.89 & 7.08 & 8.28 & 9.48 & 10.68 \\
\hline 5 & 3.85 & 4.91 & 6.07 & 7.26 & 8.46 & 9.66 & 10.86 \\
\hline 6 & 5.47 & 6.97 & 8.94 & 9.86 & 11.22 & 12.69 & 13.96 \\
\hline 7 & 5.34 & 6.90 & 8.68 & 10.50 & 12.33 & 13.78 & $*$ \\
\hline 8 & 6.96 & 8.73 & 10.42 & 12.40 & 13.47 & $*$ & $*$ \\
\hline
\end{tabular}

Table 2: Values of $\Delta$ for the methods $\mathcal{M}(k), k=2, \cdots, 8$

\section{Numerical experiments}

\subsection{Accuracy tests}

In order to numerically verify the order of methods $\mathcal{M}(k)$ we integrate the test problem proposed by Kaps [16], and used in [4] :

$$
\left\{\begin{array}{l}
y_{1}^{\prime}=-\left(2+\epsilon^{-1}\right) y_{1}+\epsilon^{-1} y_{2}^{2} \\
y_{2}^{\prime}=y_{1}-y_{2}\left(1+y_{2}\right) \\
y_{1}(0)=1 \\
y_{2}(0)=1 \\
0 \leq x \leq X
\end{array}\right.
$$

with the following exact solution for any value of $\epsilon$ :

$$
\left\{\begin{array}{l}
y_{1}=e^{-2 x} \\
y_{2}=e^{-x}
\end{array}\right.
$$

In Table 2 we have listed the values of $\Delta$, where $\Delta$ denotes the number of correct digits of the numerical solution at the end of the interval (i.e. $\left.\Delta=-\log _{10}\left(\max _{i=1,2}\left|y_{i}(X)-y_{n, i}\right|\right)\right)$. All experiments have been done with $\varepsilon=10^{-8}$ and $X=4$, in order to avoid the influence of the starting procedure (in this case, the $k$ first approximations are actually exact) ${ }^{2}$. These values have been plotted on a graph (see Figure 4), and a good adequation between theoretical and real order can be observed. However, for very high precision (more than $10^{-14}$ ), rounding errors become predominent (see Figure 4). A star * indicates unreliable results.

\subsection{Stability tests}

The stability of the methods is tested by integrating a problem in which the Jacobian matrix $J$ has purely imaginary eigenvalues :

$$
\left\{\begin{array}{l}
y_{1}^{\prime}=-\alpha y_{2}+(1+\alpha) \cos (x) \\
y_{2}^{\prime}=\alpha y_{1}-(1+\alpha) \sin (x) \\
y_{1}(0)=0 \\
y_{2}(0)=1 \\
0 \leq x \leq X
\end{array}\right.
$$

with exact solution :

$$
\left\{\begin{array}{l}
y_{1}=\sin (x) \\
y_{2}=\cos (x)
\end{array}\right.
$$

\footnotetext{
${ }^{2}$ Experiments were performed with a MATLAB written code in double precision (precision parameter $=2.10^{-16}$ )
} 


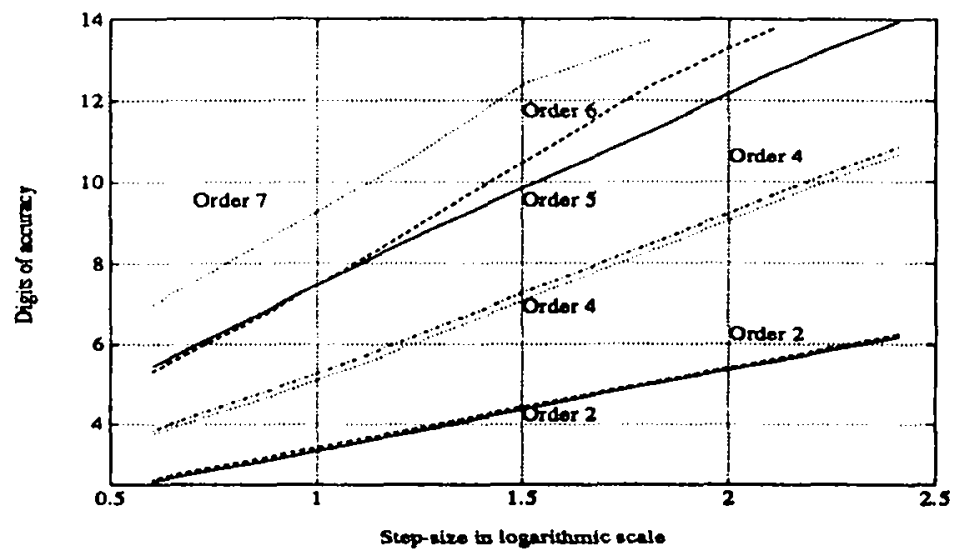

Figure 4: Error distributions for the methods $\mathcal{M}(k), k=2, \cdots, 8$ of Table (1)

for any value of the parameter $\alpha$. Therefore, $J$ has the following expression :

$$
J=\left(\begin{array}{cc}
0 & -\alpha \\
\alpha & 0
\end{array}\right)
$$

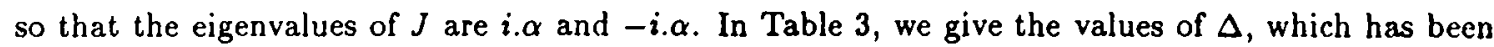
defined above, as a function of the stepsize. We finally illustrate the better behavior of methods $\mathcal{M}(k)$ over BDF's (Backward Differentiation Formulas) for orders 4, 5 and 6 . The second order BDF is indeed A-stable, whereas for orders higher than 6, the BDF's are no longer zero-stable. Overflow is indicated by $\infty$ and values of $\Delta$ corresponding to stepsizes that are theoretically unstable are underlined.

\begin{tabular}{|c|c|c|c|c|c|c|}
\hline \hline Method & $\mathrm{h}=4 / 5$ & $\mathrm{~h}=2 / 5$ & $\mathrm{~h}=1 / 5$ & $\mathrm{~h}=1 / 10$ & $\mathrm{~h}=1 / 20$ & $\mathrm{~h}=1 / 40$ \\
\hline $\mathcal{M}(2)$ & 1.43 & 1.73 & 2.05 & $2.39 \mathrm{v}$ & 2.81 & 3.33 \\
\hline \hline $\mathcal{M}(3)$ & 1.88 & 2.42 & 2.91 & 3.40 & 3.95 & 4.53 \\
\hline \hline$B D F_{4}$ & 2.2 & $\infty$ & $\infty$ & $\infty$ & $\underline{2.9}$ & $\underline{8.2}$ \\
\hline $\mathcal{M}(4)$ & 2.29 & 3.13 & 3.95 & 4.79 & 5.73 & 6.73 \\
\hline \hline $\mathcal{M}(5)$ & 2.62 & 3.81 & 4.92 & 5.88 & 6.82 & 7.66 \\
\hline \hline$\overline{B D} F_{5}$ & $\underline{-0.1}$ & $\infty$ & $\infty$ & $\infty$ & 8.5 & 10.3 \\
\hline $\mathcal{M}(6)$ & 2.93 & 4.46 & 5.87 & 7.25 & 8.74 & 9.79 \\
\hline \hline$\overline{B D} F_{6}$ & $\infty$ & $\infty$ & $\infty$ & $\infty$ & 9.64 & 11.48 \\
\hline $\mathcal{M}(7)$ & 3.20 & 5.06 & 7.03 & 8.34 & 9.62 & 10.79 \\
\hline $\mathcal{M}(8)$ & 3.45 & 5.69 & 8.31 & 9.60 & 11.73 & 13.01 \\
\hline \hline
\end{tabular}

Table 3: Values of $\Delta$ for $X=100$ and $\alpha=10$

\section{Conclusion}

In this paper, we have tried to improve the results obtained by B.P. Sommeijer, W. Couzy and P.J. van der Houwen in [4]. They introduced a class of seemingly very promising block-methods suitable for integrating ordinary differential equations on parallel computers. On a first step, we analyse 
these methods by considering them as "general linear methods". We applied the different results of Butcher and Skeel theory, and we obtained a refinement of the order conditions that gave much hope for higher order A-stable methods. On a second step, we got a practical condition for A-stability by applying well-known results of complex analysis. This led us to an "experimental" search for A-stable methods based on symbolic calculus. We got a third-order L-stable method using two processors that compares favorably with its BDF equivalent. However, even for very low order, the amount of computation soon becomes a barrier. Moreover, our criterium for A-stability does not furnish trivial indications for the choice of free coefficients. Consequently, a fruitful application of this approach to higher orders seems particulary hard and tedious (though, we are still convinced that it can give some interesting results for order 4 and maybe for order 5). That is why we turned to a more systematic search whose aim was to find a generic method with good stability properties. We have designed a new class of parallel block methods which can be very easily implemented on a parallel computer, and we have also proved that they are L-stable up to order 7. Numerical results and local analysis both indicate that these methods are likely to be L-stable for higher orders. Finally, have integrated two initial value problems in order to confirm the favourable behavior of the methods. These experiments are in total agreement with the theoretical results. So far, we have constructed a class of methods with the same stability properties as the Gauss-Lobatto Runge-Kutta methods, but which are far less complex with respect to implementation. More precisely, the $(s . m)^{\text {th }}$-order system 3 of Gauss-Lobatto methods ( $s$ the stage number) has been transformed into $s$ systems of order $m$ which can be solved simultaneously. This should breed very substantial speedups on a parallel computer.

\section{Acknowledgements}

We would like to thank Michel Crouzeix who provided us with numerous ideas and therefore brought an important contribution to this paper.

\section{A $\mathcal{M}(2)$ with $r=4$}

$$
A=\left(\begin{array}{cc}
1 / 2 & 1 / 2 \\
-1 / 4 & 5 / 4
\end{array}\right), \quad D=\left(\begin{array}{cc}
1 / 2 & 0 \\
0 & 3 / 4
\end{array}\right), E=\left(\begin{array}{c}
1 / 2 \\
1 / 6
\end{array}\right)
$$

We have here $C_{2}=(1 / 2,1 / 4)^{T}$ and :

$$
\frac{Q(A)}{Q(1)}=\left(\begin{array}{ll}
-1 & 2 \\
-1 & 2
\end{array}\right) \Longrightarrow \mathbf{E} C_{2}=0
$$

so that the method is of order 2 .

B $\mathcal{M}(3)$ with $r=11 / 2$

$$
A=\left(\begin{array}{ccc}
2 / 11 & 1 & -2 / 11 \\
-3 / 11 & \frac{12}{11} & 2 / 11 \\
-1 / 11 & -1 / 11 & \frac{13}{11}
\end{array}\right), D=\left(\begin{array}{ccc}
4 / 11 & 0 & 0 \\
0 & \frac{6}{11} & 0 \\
0 & 0 & \frac{8}{11}
\end{array}\right), \quad E=\left(\begin{array}{c}
-1 / 6 \\
1 / 3 \\
\frac{11}{24}
\end{array}\right)
$$

The equation $\mathbf{E}(r) C_{3}(r)=0$ has the following expression :

$$
6 r^{3}-63 r^{2}+185 r-144=0
$$

\footnotetext{
${ }^{3}$ Each new approximation of the solution requires the solution of an implicit system.
} 
It has no rational solution. However, $r=3.184850644$ "seems" to lead to a third-order L-stable method.

C $\mathcal{M}(4)$ with $r=5$

$$
A=\left(\begin{array}{cccc}
2 / 15 & 6 / 5 & -2 / 5 & 1 / 15 \\
-1 / 10 & 3 / 5 & \frac{7}{10} & -1 / 5 \\
\frac{4}{15} & -6 / 5 & \frac{12}{5} & -\frac{7}{15} \\
5 / 6 & -3 & 7 / 2 & -1 / 3
\end{array}\right), D=\left(\begin{array}{cccc}
2 / 5 & 0 & 0 & 0 \\
0 & 3 / 5 & 0 & 0 \\
0 & 0 & 4 / 5 & 0 \\
0 & 0 & 0 & 1
\end{array}\right), E=\left(\begin{array}{c}
1 / 12 \\
-1 / 12 \\
1 / 4 \\
\frac{13}{12}
\end{array}\right)
$$

We have here $C_{4}=(4 / 5,-6 / 5,24 / 5,26)^{T}$ and :

$$
\frac{Q(A)}{Q(1)}=\left(\begin{array}{llll}
\frac{35}{36} & -\frac{25}{6} & \frac{65}{12} & -\frac{11}{9} \\
\frac{35}{96} & -\frac{25}{6} & \frac{65}{12} & -\frac{11}{9} \\
\frac{36}{36} & -\frac{25}{6} & \frac{65}{12} & -\frac{11}{9} \\
\frac{35}{36} & -\frac{25}{6} & \frac{65}{12} & -\frac{11}{9}
\end{array}\right) \Rightarrow E C_{4}=0
$$

so that $\mathcal{M}(4)$ is of order 4 .

D $\mathcal{M}(5)$ with $r=6$

$A=\left(\begin{array}{ccccc}1 / 12 & \frac{23}{18} & -1 / 2 & 1 / 6 & -1 / 36 \\ -1 / 24 & 1 / 3 & 1 & -1 / 3 & 1 / 24 \\ 1 / 18 & -1 / 3 & 1 & 4 / 9 & -1 / 6 \\ -\frac{5}{24} & \frac{10}{9} & -5 / 2 & 10 / 3 & -\frac{53}{22} \\ -\frac{13}{12} & \frac{31}{6} & -19 / 2 & \frac{47}{6} & -\frac{17}{12}\end{array}\right), D=\left(\begin{array}{ccccc}1 / 3 & 0 & 0 & 0 & 0 \\ 0 & 1 / 2 & 0 & 0 & 0 \\ 0 & 0 & 2 / 3 & 0 & 0 \\ 0 & 0 & 0 & 5 / 6 & 0 \\ 0 & 0 & 0 & 0 & 1\end{array}\right), \quad E=\left(\begin{array}{c}-1 / 20 \\ 1 / 30 \\ -1 / 20 \\ 1 / 5 \\ \frac{77}{60}\end{array}\right)$

The equation $\mathbb{E}(r) C_{5}(r)=0$ is here :

$$
120 r^{5}-3000 r^{4}+26650 r^{3}-102850 r^{2}+166904 r-86400=0
$$

E $\mathcal{M}(6)$ with $r=6$

$$
\begin{gathered}
A=\left(\begin{array}{cccccc}
1 / 15 & \frac{49}{36} & -2 / 3 & 1 / 3 & -1 / 9 & \frac{1}{60} \\
-1 / 40 & 1 / 4 & 7 / 6 & -1 / 2 & 1 / 8 & -\frac{1}{60} \\
1 / 45 & -1 / 6 & 2 / 3 & \frac{7}{9} & -1 / 3 & 1 / 30 \\
-1 / 24 & \frac{3}{18} & -5 / 6 & 5 / 3 & \frac{7}{72} & -1 / 6 \\
1 / 5 & -5 / 4 & 10 / 3 & -5 & 5 & -\frac{77}{60} \\
\frac{599}{360} & -\frac{39}{4} & \frac{47}{2} & -\frac{529}{18} & \frac{153}{8} & -\frac{83}{20}
\end{array}\right),\left(\begin{array}{cccccc}
1 / 3 & 0 & 0 & 0 & 0 & 0 \\
0 & 1 / 2 & 0 & 0 & 0 & 0 \\
0 & 0 & 2 / 3 & 0 & 0 & 0 \\
0 & 0 & 0 & 5 / 6 & 0 & 0 \\
0 & 0 & 0 & 0 & 1 & 0 \\
0 & 0 & 0 & 0 & 0 & 7 / 6
\end{array}\right) \\
E=\left(\begin{array}{c}
1 / 30 \\
-\frac{1}{60} \\
\frac{1}{60} \\
-1 / 30 \\
1 / 6 \\
\frac{223}{140}
\end{array}\right)
\end{gathered}
$$

The equation $\mathrm{E}(r) C_{6}(r)=0$ has the form:

$$
\begin{array}{r}
360 r^{6}-12420 r^{5}+161250 r^{4}-992475 r^{3} \\
+2987647 r^{2}-4037154 r+1814400=0
\end{array}
$$




\section{F $\quad \mathcal{M}(7)$ with $r=6$}

$$
\begin{aligned}
& A=\left(\begin{array}{ccccccc}
1 / 18 & \frac{257}{180} & -5 / 6 & 5 / 9 & -\frac{5}{18} & 1 / 12 & -\frac{1}{90} \\
-\frac{1}{61} & 1 / 5 & \frac{31}{24} & -2 / 3 & 1 / 4 & -1 / 15 & \frac{1}{120} \\
\frac{1}{90} & -1 / 10 & 1 / 2 & 1 & -1 / 2 & 1 / 10 & -\frac{1}{90} \\
-\frac{1}{72} & 1 / 9 & -\frac{5}{12} & \frac{10}{9} & \frac{37}{72} & -1 / 3 & 1 / 36 \\
1 / 30 & -1 / 4 & 5 / 6 & -5 / 3 & 5 / 2 & -\frac{17}{60} & -1 / 6 \\
-\frac{7}{36} & 7 / 5 & -\frac{35}{8} & \frac{70}{2} & -\frac{35}{6} & 7 & -\frac{223}{120} \\
-\frac{34}{15} & \frac{7114}{45} & -46 & \frac{223}{3} & -\frac{634}{9} & \frac{188}{5} & -\frac{18}{15}
\end{array}\right) \\
& D=\left(\begin{array}{ccccccc}
1 / 3 & 0 & 0 & 0 & 0 & 0 & 0 \\
0 & 1 / 2 & 0 & 0 & 0 & 0 & 0 \\
0 & 0 & 2 / 3 & 0 & 0 & 0 & 0 \\
0 & 0 & 0 & 5 / 6 & 0 & 0 & 0 \\
0 & 0 & 0 & 0 & 1 & 0 & 0 \\
0 & 0 & 0 & 0 & 0 & 7 / 6 & 0 \\
0 & 0 & 0 & 0 & 0 & 0 & 4 / 3
\end{array}\right), E=\left(\begin{array}{c}
-1 / 42 \\
\frac{1}{105} \\
-\frac{1}{140} \\
\frac{1}{105} \\
-1 / 42 \\
1 / 7 \\
\frac{129}{70}
\end{array}\right)
\end{aligned}
$$

As before, to get one order higher, $r$ should satisfy :

$$
\begin{array}{r}
-87442264 r^{3}-254393394 r+18305175 r^{4}+218041817 r^{2}-2044770 r^{5} \\
+101606400+114660 r^{6}-2520 r^{7}=0
\end{array}
$$

$r=6.107690185$ is likely to lead to an $7^{\text {th }}$-order L-stable method.

G $\mathcal{M}(8)$ with $r=7$

$$
\begin{aligned}
& A=\left(\begin{array}{cccccccc}
\frac{2}{49} & \frac{99}{70} & -6 / 7 & 5 / 7 & -\frac{10}{21} & 3 / 14 & -\frac{2}{35} & \frac{1}{147} \\
-\frac{1}{98} & 1 / 7 & \frac{187}{140} & -5 / 7 & \frac{5}{14} & -1 / 7 & 1 / 28 & -\frac{1}{245} \\
\frac{1}{735} & -\frac{2}{35} & \frac{12}{35} & \frac{8}{7} & -4 / 7 & \frac{6}{35} & -\frac{4}{105} & \frac{1}{245} \\
-\frac{1}{196} & 1 / 21 & -3 / 14 & 5 / 7 & \frac{23}{28} & -3 / 7 & 1 / 14 & -\frac{1}{147} \\
\frac{2}{245} & -1 / 14 & 2 / 7 & -5 / 7 & \frac{10}{7} & \frac{23}{70} & -2 / 7 & 1 / 49 \\
-1 / 42 & 1 / 5 & -3 / 4 & 5 / 3 & -5 / 2 & 3 & -\frac{9}{20} & -1 / 7 \\
\frac{8}{49} & -4 / 3 & \frac{24}{5} & -10 & \frac{40}{3} & -12 & 8 & -\frac{481}{245} \\
\frac{2287}{980} & -\frac{647}{35} & \frac{4469}{70} & -\frac{877}{7} & \frac{4259}{28} & -\frac{4049}{35} & \frac{3629}{70} & -\frac{3669}{245}
\end{array}\right) \\
& D=\left(\begin{array}{cccccccc}
2 / 7 & 0 & 0 & 0 & 0 & 0 & 0 & 0 \\
0 & 3 / 7 & 0 & 0 & 0 & 0 & 0 & 0 \\
0 & 0 & 4 / 7 & 0 & 0 & 0 & 0 & 0 \\
0 & 0 & 0 & 5 / 7 & 0 & 0 & 0 & 0 \\
0 & 0 & 0 & 0 & 6 / 7 & 0 & 0 & 0 \\
0 & 0 & 0 & 0 & 0 & 1 & 0 & 0 \\
0 & 0 & 0 & 0 & 0 & 0 & \frac{8}{7} & 0 \\
0 & 0 & 0 & 0 & 0 & 0 & 0 & \frac{9}{7}
\end{array}\right), E=\left(\begin{array}{c}
\frac{1}{56} \\
-\frac{1}{168} \\
\frac{1}{280} \\
-\frac{1}{180} \\
\frac{1}{168} \\
\frac{1}{56} \\
1 / 8 \\
\frac{4889}{2520}
\end{array}\right)
\end{aligned}
$$

For sake of completness, we give the condition on $r$ for order 8 :

$$
\begin{array}{r}
2289061754 r^{3}+5066650482 r-580819883 r^{4}-4907763545 r^{2}+83919360 r^{5} \\
-6850200 r^{6}-1828915200+292320 r^{7}-5040 r^{8}=0
\end{array}
$$




\section{Root locus curves}

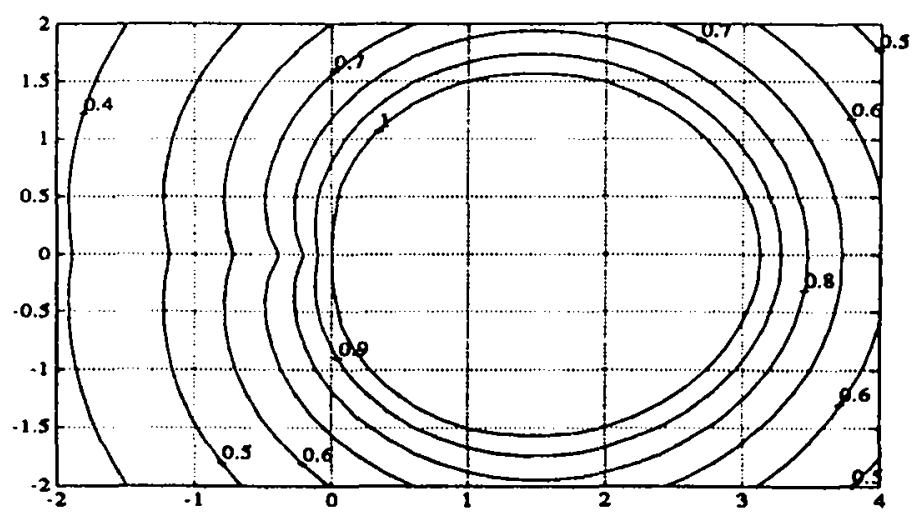

Figure 5: Root locus curve for $\mathcal{M}(2)$ with $r=4$

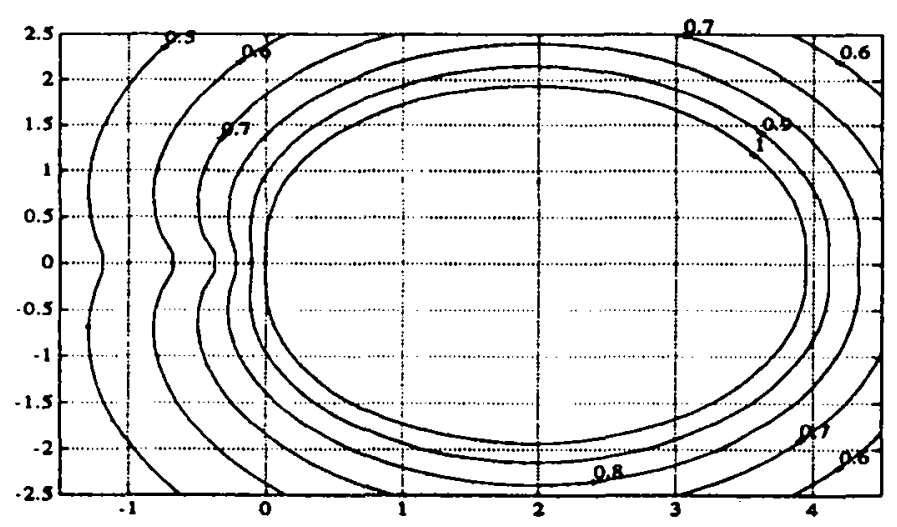

Figure 6: Root locus curve for $\mathcal{M}(3)$ with $r=11 / 2$ 


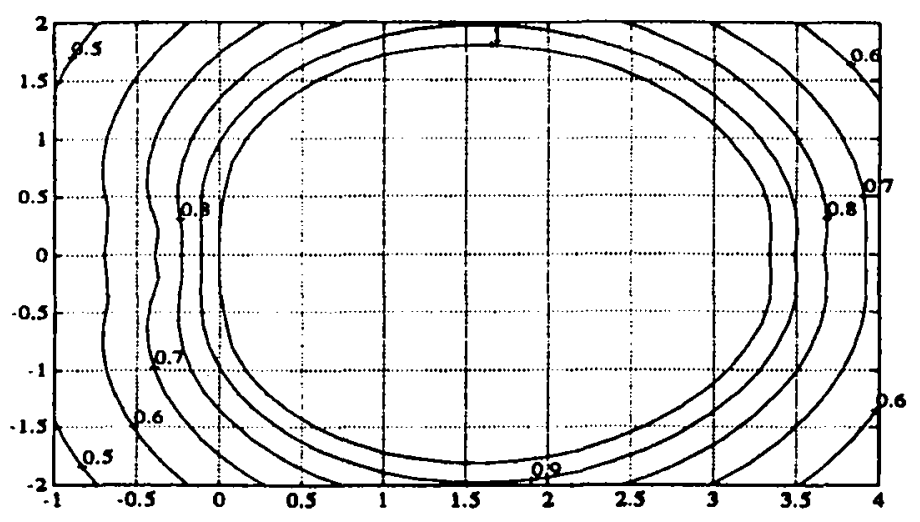

Figure 7: Root locus curve for $\mathcal{M}(4)$ with $r=5$

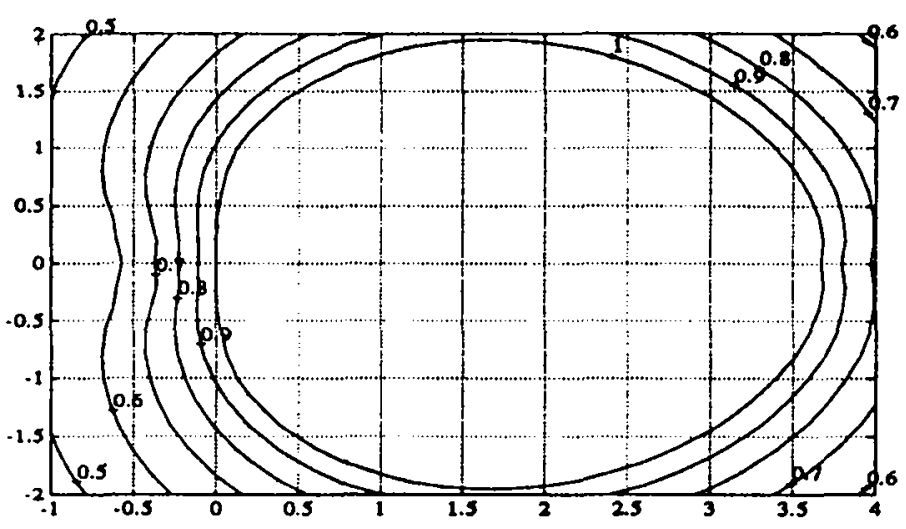

Figure 8: Root locus curve for $\mathcal{M}(5)$ with $r=6$

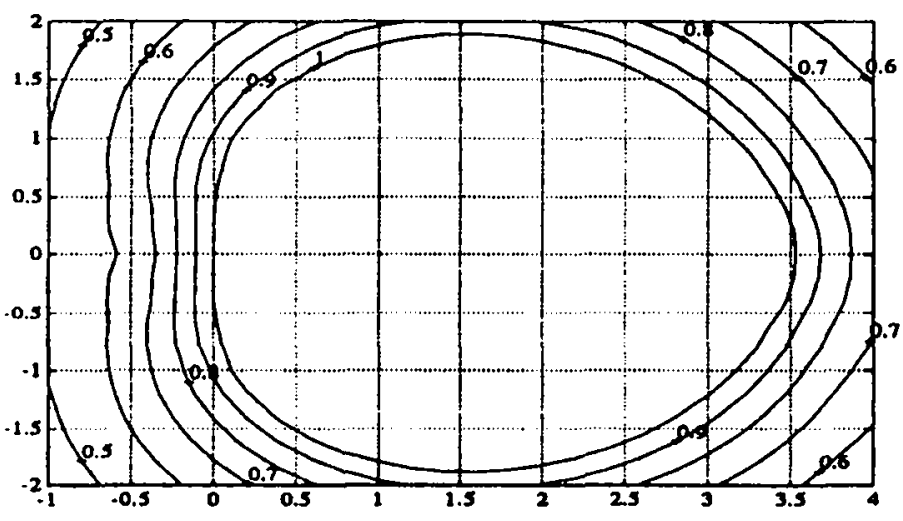

Figure 9: Root locus curve for $\mathcal{M}(6)$ with $r=6$ 


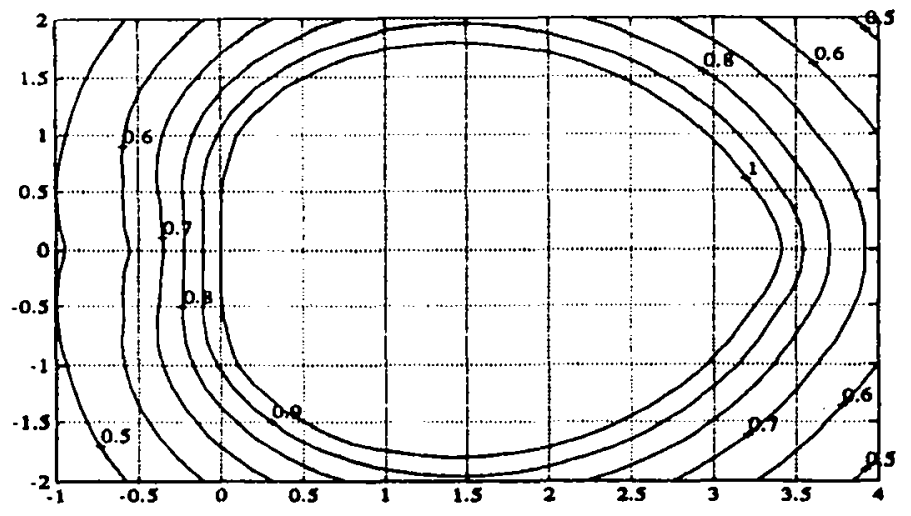

Figure 10: Root locus curve for $\mathcal{M}(7)$ with $r=6$

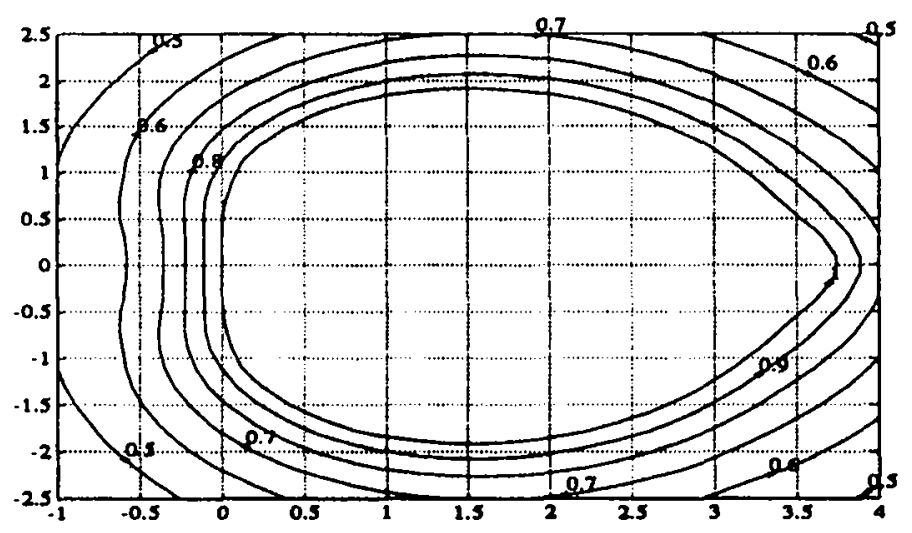

Figure 11: Root locus curve for $\mathcal{M}(8)$ with $r=7$ 


\section{References}

[1] E. Hairer, S.P. Norsett and G. Wanner Solving Ordinary Differential Equations I. Nonstiff Problems (Springer, Berlin, 1987)

[2] E. Hairer and G. Wanner Solving Ordinary Differential Equations II. Stiff and Differential. Algebraic Problems (Springer, Berlin, 1991)

[3] J.C. Butcher The numerical analysis of ordinary differential equations. Runge-Kutta and general linear methods (John Wiley and Sons, 1987)

[4] B.P. Sommeijer, W. Couzy and P.J. van der Houwen A-Stable Parallel Block Methods (Research Report 1989)

[5] C.W. Gear Parallel methods for ordinary differential equations (Report R-87-1360, Departement of Computer Science, University of Illinois, Urbana, IL, 1986

[6] W.H. Enright Optimal second derivative methods for stiff systems (In Stiff Differential Systems, ed. by R.A. Willoughby, Plenum Press, New York, 1974)

[7] W.H. Enright Second derivative multistep methods for stiff ordinary differential equations (SIAM J. Numer. Anal., vol. 11, pp. 321-331, 1974)

[8] A. Bellen and M. Zennaro Parallel Algorithms for Initial Value Problems for Difference and Differential Equations (J. Comput. Appl. Math. 25, pp 341-350, 1989

[9] R. Vermiglio Parallel Step Methods for Difference and Differential Equations on a hypercube, (C.N.R. Progetto Finaizzato "Sistemi Informatici e Calcolo Parallelo", Tech. Rep. 1/1)

[10] I. Lie and S.P. Norsett Superconvergence for multistep collocation (Math. of Comput., vol. 57. pp. 65-79, 1989)

[11] P. Chartier Application of Bellen's method to ODE's with dissipative right-hand side (to appear in Proceedings of the $10^{\text {th }}$ International Conference on Comp. Meth. in Appl. Sciences and Enginnering, 1992)

[12] J.R. Cash On the integration of stiff sytems of ODEs using extended backward differentiation formulae (Numer. Math., vol. 34, pp. 235-246, 1980)

[13] J.R. Cash Second derivative extended backward differentiation formulas for the numerical integration of stiff systems (SIAM J. Numer. Anal., vol. 18, 00. 21-36, 1981)

[14] M.T. Chu and H. Hamilton Parallel solution of ODEs by multi-block methods (SIAM J. Sci. Stat. Comp., vol. 8, pp. 342-353, 1987)

[15] L.F. Shampine and H.A. Watts A-stable implicit one-step methods (BIT, vol. 12, pp. 252-266, 1972)

[16] P. Kaps Rosenbrock-type methods (Numerical methods for stiff initial value problems, eds.: G. Dahlquist and R. Jeltsch, Bericht Nr.9, Inst. fur Geometrie und Praktische Mathematik der RWTH Aachen) 


\section{LISTE DES PUBLICATIONS INTERNES IRISA 1992}

PI 624 SIGNAL AS A MODEL FOR REAL-TIME AND HYBRID SYSTEMS

Albert BENVENISTE, Michel LE BORGNE, Paul LE GUERNIC

Janvier 1992, 22 pages.

PI 625 ON THE CENTRAL-LIMIT THEOREM FOR TRACKING ESTIMATORS WITH SMALL GAIN - INFINITE HORIZON CASE

Bernard DELYON, Anatoli JUDITSKY

Janvier 1992, 16 pages.

PI 626 A MONTE CARLO METHOD BASED ON ANTITHETIC VARIATES FOR NETWORK RELIABILITY COMPUTATIONS

Mohamed EL KHADIRI, Gerardo RUBINO

Janvier 1992, 28 pages.

PI 627 CONSTRAINED MULTISCALE MARKOV RANDOM FIELDS AND THE ANALYSIS OF VISUAL MOTION

Fabrice HEITZ, Patrick PEREZ, Patrick BOUTHEMY

Janvier 1992, 40 pages.

PI 628 ON ITERATIVE REFINEMENT FOR THE SPECTRAL DECOMPOSITION OF SYMMETRIC MATRICES

Alexander N. MALYSHEV

Janvier 1992, 26 pages.

PI 629 STRUCTURAL OPERATIONAL SPECIFICATIONS AND TRACE AUTOMATA Eric BADOUEL, Philippe DARONDEAU

Janvier 1992, 36 pages.

PI 630 EREBUS, A DEBUGGER FOR ASYNCHRONOUS DISTRIBUTED COMPUTING SYSTEM

Michel HURFIN, Noël PLOUZEAU, Michel RAYNAL

Janvier 1992, 14 pages.

PI 631 PROTOCOLES SIMPLES POUR L'IMPLEMENTATION REPARTIE DES SEMAPHORES

Michel RAYNAL

Janvier 1992, 14 pages.

PI 632 L-STABLE PARALLEL ONE-BLOCK METHODS FOR ORDINARY DIFFERENTIAL EQUATIONS

Philippe CHARTIER, Bernard PHILIPPE

Janvier 1992, 28 pages.

PI 633 ON EFFICIENT CHARACTERIZING SOLUTIONS OF LINEAR DIOPHANTINE EQUATIONS AND ITS APPLICATION TO DATA DEPENDENCE ANALYSIS Christine EISENBEIS, Olivier TEMAM, Harry WIJSHOFF Janvier 1992, 22 pages.

PI 634 UN NOYAU DE SYSTEME REPARTI POUR LES APPLICATIONS GEREES PAR UN TEMPS VIRTUEL

Janvier 1992, 20 pages. 
ISSN 0249-6399 